ISSN: 2215-2644

revedu@gmail.com

Universidad de Costa Rica

Costa Rica

\title{
El portafolio electrónico como instrumento de evaluación y como estrategia de autorregulación en la formación de profesionales en música
}

\author{
Capistrán Gracia[1], Raul Wenceslao \\ El portafolio electrónico como instrumento de evaluación y como estrategia de autorregulación en la formación de \\ profesionales en música \\ Revista Educación, vol. 42, núm. 2, 2018 \\ Universidad de Costa Rica, Costa Rica \\ Disponible en: http://www.redalyc.org/articulo. $0 a ? \mathrm{id}=44055139019$ \\ DOI: https://doi.org/10.15517/revedu.v42i2.25937
}

Esta obra está bajo una Licencia Creative Commons Atribución-NoComercial-SinDerivar 3.0 Internacional. 


\title{
El portafolio electrónico como instrumento de evaluación y como estrategia de autorregulación en la formación de profesionales en música
}

\author{
The Electronic Portfolios as an Assessment Tool and as Self-regulation Strategy in the Formation of Professional \\ Musicians
}

Raul Wenceslao Capistrán Gracia[1]

Universidad Autónoma de Aguascalientes, México

raul_capistran@hotmail.com

(iD http://orcid.org/0000-0002-4594-8570

\author{
DOI: https://doi.org/10.15517/revedu.v42i2.25937
}

Redalyc: http://www.redalyc.org/articulo.oa?id=44055139019

Recepción: 17 Agosto 2016

Aprobación: 03 Agosto 2018

\section{ReSUMEN:}

El estudiantado de música requiere de estrategias de autoevaluación no solo para determinar si ha mejorado, sino también para seleccionar las estrategias de práctica más adecuadas en caso de que no haya mejoría y lograr vencer los retos técnico-musicales. Por su parte, el personal docente a cargo debe contar con instrumentos de evaluación válidos y confiables que le permitan llevar a cabo una valoración objetiva, realista y justa del desempeño estudiantil. En este artículo, el autor ofrece una revisión a profundidad de la bibliografía sobre cuatro estrategias de autoevaluación de alto nivel metacognitivo y propone el uso del portafolio electrónico desde dos perspectivas: a) como estrategia de autorregulación que ayude al estudiantado a asumir una mayor responsabilidad por su propio aprendizaje, al monitorizar y autoevaluar sus esfuerzos a través de la recopilación de evidencias que le permiten reflexionar y tener una percepción más realista de sus logros; y b) como instrumento que contribuye para que el maestro o maestra lleve a cabo una evaluación más integral de los avances estudiantiles. Se espera que la información que aquí se proporciona fomente una práctica musical más holística y efectiva, y una evaluación más integral.

Palabras Clave: Portafolio electrónico, evaluación, autoevaluación, autorregulación, práctica musical.

\section{Abstract:}

Music students require self-assessment strategies not only to determine if they have improved, but also to select the most appropriate practice strategies in case there is no improvement in order to overcome technical-musical challenges. On the other hand, teachers must have valid and reliable assessment instruments that allow them to carry out an objective, realistic and fair assessment of student performance. In this article, the author offers an in-depth literature review of four metacognitive high-level self-assessment strategies and proposes the use of the electronic portfolio from two perspectives: a) as a self-regulation strategy that help students to take greater responsibility for their own learning by monitoring and self-assessing their efforts through the collection of evidence that allows them to reflect and have a more realistic perception of their achievements; and, b) as an instrument that helps teachers to carry out a more comprehensive assessment of the student's progress. It is expected that the information provided here will foster a more holistic and effective musical practice and a more integral assessment.

KEYWORDS: electronic portfolio, assessment, self-assessment, self-regulation, musical practice.

\section{INTRODUCCIÓN}

Después de su lección de instrumento, cada estudiante debe practicar sus ejercicios y piezas lejos de la supervisión de su maestro o maestra, sin que este personal tenga idea de lo que pasa en el cubículo de práctica (Gaunt, 2008; Jorgensen, 2000; Sloboda, Davidson, Howe y Moore, 1996). Durante ese tiempo, debe tomar decisiones de vital importancia sobre cómo organizar su práctica, que estrategias utilizar para dominar los retos técnicos-musicales y cómo autoevaluar los resultados obtenidos (Byo y Cassidy, 2008).

Por lo anterior, se vuelve imperativo que el estudiantado desarrolle habilidades metacognitivas, es decir, que aprenda a aprender por sí mismo, para que pueda regular su propia práctica, identificar los desafíos, buscar estrategias adecuadas para hacer frente a problemas específicos, autoevaluarse y adaptar o modificar su ejecución, a fin de poder mejorar (Boucher, Dube y Creech, 2017; Hallam, 2001; Jorgensen, 2000 y 2004). 
Esta última fase, la de la autoevaluación, es quizá la más importante, dado que establece las bases para el reinicio del ciclo de aprendizaje: planeación de la práctica - ejecución de la práctica - autoevaluación de la práctica (Jorgensen, 2004).

Desgraciadamente, en muchas ocasiones es posible escuchar estudiantes practicando de una misma manera, generalmente, repitiendo la pieza una y otra vez de principio a fin, a pesar de que es evidente que no están obteniendo los resultados esperados (Capistrán, 2014; Pitts, Davidson y McPherson, 2000). Por lo anterior, varias investigaciones han enfatizado la importancia de que los cuerpos docentes fomenten en sus estudiantes el desarrollo de habilidades para autoevaluarse, a fin de garantizar que tengan una mejora sostenida en sus lecciones de instrumento (Boucher et al., 2017; Hallam, 1997, 2001; McPherson y Renwick, 2001; Pitts, Davidson y McPherson, 2000). En conclusión, el estudiantado de música requiere de estrategias de autoevaluación confiables que le permitan determinar si ha mejorado en su ejecución para que, sobre la base de la reflexión y el análisis, pueda seleccionar la manera de practicar más adecuada en caso de que no haya mejoría y logre vencer los retos técnico-musicales (Hallam, 2001; Jorgensen, 2004; Lundin, 1967; McPherson y McCormick, 1999; Nielsen, 1999; Rosenthal, Durairaj y Magann, 2009; Zimmerman, 2008).

En este artículo, el autor proporciona una revisión bibliográfica a profundidad sobre cuatro estrategias de autoevaluación de alto nivel metacognitivo, las cuales podrían ser de gran utilidad para estudiantes y docentes, y propone el uso del portafolio electrónico desde dos perspectivas: a) como estrategia de autorregulación que fomenta en el estudiantado la responsabilidad por su propio aprendizaje, al monitorizar y evaluar sus propios esfuerzos por medio de la reunión de evidencias y de su comparación, análisis y reflexión correspondiente; y, b) como instrumento de evaluación por medio del cual se reúnen productos que reflejan los esfuerzos, avances y logros y que permiten una evaluación integral, objetiva y justa por parte del personal docente.

El autor espera que la información contenida en este artículo sea de utilidad para estudiantes, maestras y maestros en el desarrollo de una práctica musical más efectiva y satisfactoria.

\section{La audiograbación, la videograbación, la evaluación de pares y el uso de listas de cotejo. Revisión bibliográfica}

Esta revisión tiene como finalidad dar un panorama de los resultados de investigaciones empíricas que avalan el nivel de utilidad y efectividad de cuatro estrategias de autoevaluación de alto nivel metacognitivo utilizadas en la práctica musical: la audio grabación, la video grabación, la evaluación de pares y el uso de listas de cotejo.

La búsqueda de información se llevó a cabo a través de la exploración en bases de datos bibliográficos tales como Ebsco Host, Jstor, Dialnet, Latindex, Scopus, Cambridge Journals, Oxford Journals, Sage Journals, ProQuest Thesis and Dissertations y otras similares. Se consultaron artículos en revistas arbitradas e indexadas y de divulgación, libros, capítulos de libros, tesis y disertaciones cuyos contenidos, dados los exigentes protocolos de publicación, pueden ser considerados como de alto rigor académico. Para la búsqueda se utilizaron palabras clave tales como estrategias de autoevaluación musical, evaluación musical, práctica musical efectiva, rendición de cuentas, y otras similares.

\section{A) La audio-grabación}

Para Zimmerman (1986), autoevaluarse involucra llevar a cabo una comparación sistemática entre el desempeño personal y un criterio preestablecido. Estos criterios pueden ser temporales (ej. Disminuir la velocidad de una pieza para mejorar la articulación) o absolutos (ej. Tocar la obra a la velocidad establecida por el compositor o compositora). También pueden fundamentarse en el conocimiento de los estándares (ej. Afinar correctamente las notas de un pasaje musical) o en el objetivo establecido para ese momento (ej. Memorizar un fragmento de una obra). 
Se esperaría que, el estudiantado ejerciera una autoevaluación continua de su práctica sobre la base de sus conocimientos y de estándares y criterios preestablecidos. Sin embargo, los estudios indican que gran cantidad de estudiantes podría no estar consciente de sus propios errores o fallas, hasta que se confrona con su propia ejecución (Daniel, 2001; Garrison, 2009, 2010; Silveira y Gavin, 2015). Dada la complejidad que una ejecución musical entraña, no es de sorprender que la mayoría de estudiantes no esté consciente de muchos de los errores que está cometiendo o ignore las carencias o debilidades de su ejecución, ya que esta involucra una gran variedad de factores que ocurren de manera simultánea en un breve periodo. Precisión rítmica, articulación, afinación, fraseo, agógica y dinámica son solo algunos de esos factores (Capistrán y Correa, 2017). Por lo anterior, la audio-grabación se constituye en un instrumento excelente para la autoevaluación de cada estudiante, ya que permite que pueda analizar en detalle los diversos factores involucrados en su interpretación y tenga una percepción realista y objetiva de los resultados logrados (Burrack, 2002; Garrison, 2010).

En ese sentido, un estudio en el que participaron 112 estudiantes de secundaria, llevado a cabo por Silveira y Gavin (2015), reveló que la percepción estudiantil respecto a la calidad de su propia ejecución cambiaba en tres autoevaluaciones consecutivas. La primera autoevaluación fue realizada inmediatamente después de su propia ejecución, la segunda la llevaron a cabo escuchando una audio-grabación y la tercera fue realizada dos días después durante los cuales escucharon la misma audio-grabación. La investigación encontró que el criterio de los grupos participantes se agudizaba en cada evaluación y que estos valoraban su propia interpretación de una manera más estricta, por lo que reducían las calificaciones que se habían otorgado en el primer intento.

En la actualidad, la mayoría de estudiantes cuenta con teléfonos celulares que incluyen aplicaciones de audio y video que les permitirían auto-grabarse para evaluar los resultados de sus esfuerzos. Mas aun, existen aplicaciones gratuitas como Audacity, que permiten que cada estudiante, literalmente, pueda ver los cambios/ avances logrados en su ejecución musical. Por ejemplo, un crescendo o un diminuendo, un contraste entre piano y forte o una serie de dinámicas en "terrazas" pueden ser analizadas gráficamente y constituirse en medio de contraste y, en su caso, en evidencia de los logros obtenidos. Su utilidad es todavía mayor, cuando esta persona es reacia a aceptar, por ejemplo, que sus dinámicas no son del todo perceptibles.

Las Figuras1, 2 y 3 ilustran dos interpretaciones contrastantes de un mismo pasaje (los primeros 9 compases del Concierto para Piano n.o 2 op.18 de Sergei Rachmaninoff). La primera presenta el fragmento de la partitura. La segunda, refleja la ausencia total del "poco a poco crescendo", indicado en la partitura; mientras que en la tercera puede observarse claramente.

FIGURA 1

Primeros 9 compases del Concierto para Piano n.o 2 op. 18 de

Sergei Rachmaninoff. Imagen tomada de la partitura en IMSLP.

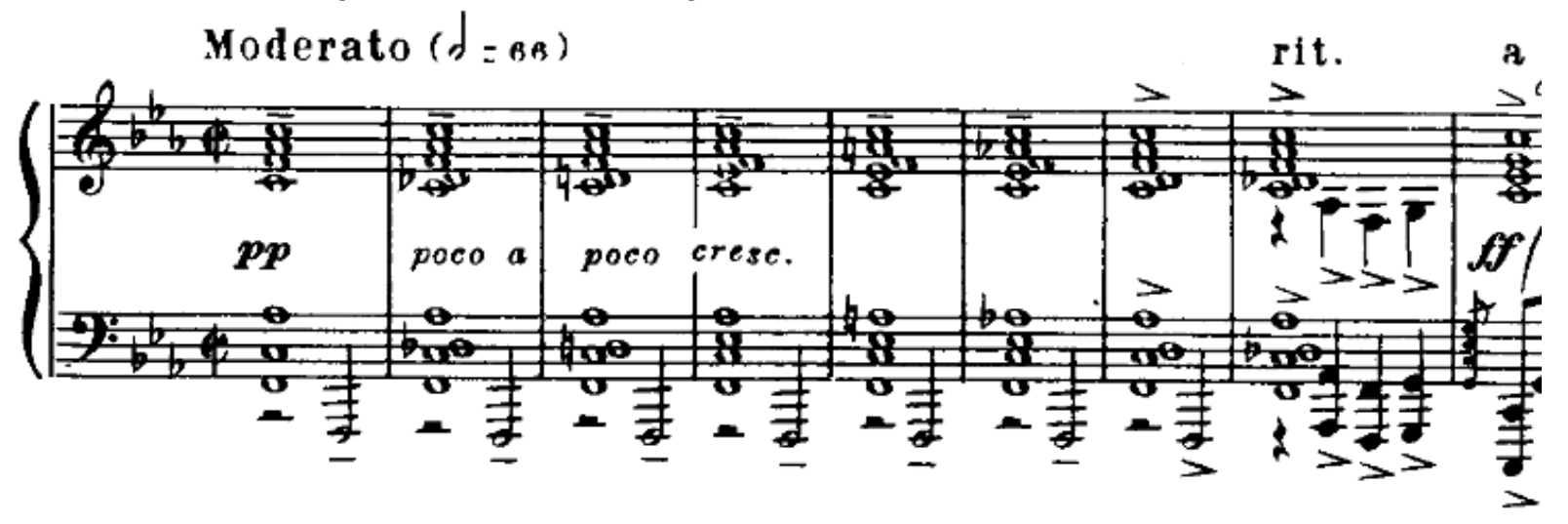

Sergei Rachmaninoff 
FIGURA 2

Gráfica en Audacity de los primeros 9 compases del Concierto para

Piano n.o 2 op.18 de Sergei Rachmaninoff en la que se muestra una dinámica plana sin el "poco a poco crescendo" indicado en la partitura.

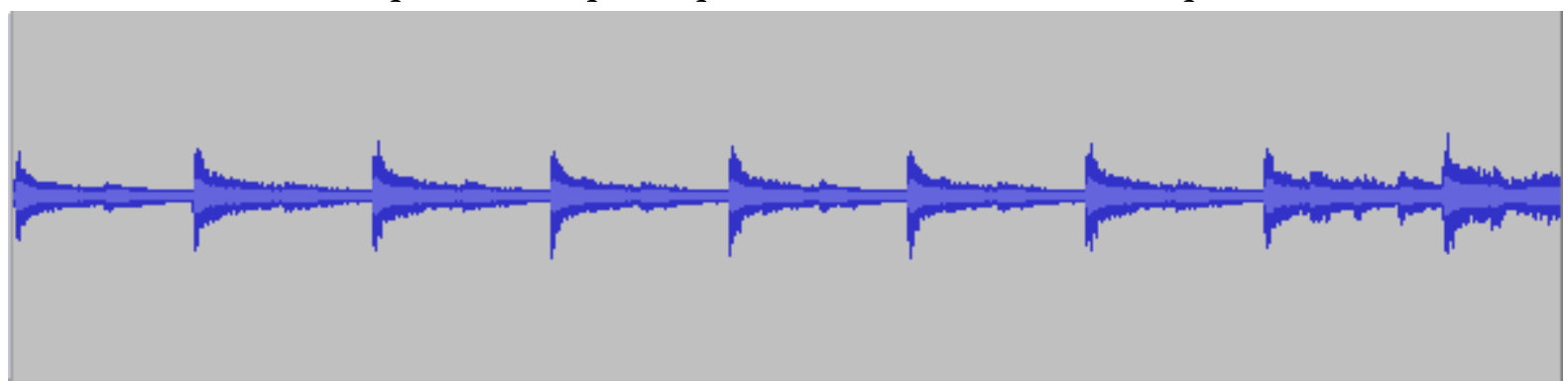

Sergei Rachmaninoff

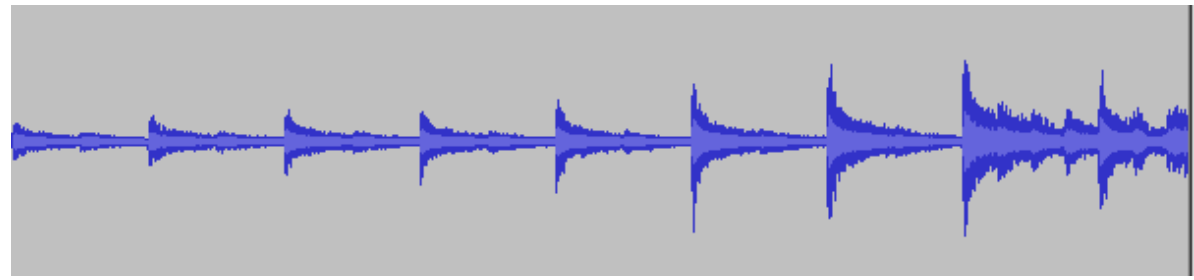

FIGURA 3

Gráfica en Audacity de los primeros 9 compases del Concierto para Piano n.o 2 op. 18 de Sergei Rachmaninoff en la que se muestra el "poco a poco crescendo" indicado en la partitura.

Sergei Rachmaninoff

Es importante mencionar que el estudiantado, sobre todo aquel que tiene poca experiencia, podría no saber exactamente qué evaluar al tratar de escuchar analíticamente una grabación. En ese sentido, su docente, en colaboración con su estudiante, puede y debe contribuir activamente al establecer con claridad las distintas dimensiones a evaluar y definir los parámetros para hacerlo. Por supuesto, el maestro o maestra puede crear una rúbrica que ayude a su estudiante a llevar a cabo una autoevaluación de manera más consciente, clara y organizada.

Aún cuando el uso de la audiograbación como herramienta para autoevaluarse ejerce un tremendo impacto en el desarrollo del pensamiento crítico y la sensibilidad, esta estrategia no es tan utilizada como podría creerse. Para McPherson y Zimmerman (2002), "La auto-grabación es una manera efectiva de monitorizar el progreso, sin embargo, es raramente usado por los músicos” (p. 342). No obstante la anterior aseveración, en los últimos años parece haber un incremento en su uso, pues personal pedagogo como Garrison (2009, 2010) afirma que cada día son más quienes hacen uso de esta importante herramienta.

\section{B) La videograbación}

Al igual que la audio-grabación, la videograbación es una herramienta que pone al alcance del estudiantado evidencias reales sobre las que puede fundamentar una autoevaluación clara y objetiva de los resultados de sus esfuerzos. Adicionalmente, la videograbación le permite evaluar aspectos de tipo técnico (postura, movimientos del torso, brazos y manos) que pudieran interferir con el logro de resultados óptimos, e incluso, puede apreciar aspectos relacionados con su desempeño en el escenario. Algunas investigaciones como la de Fautley (2013) ponderan la autoevaluación sobre la base de la videograbación, debido a que favorece que el estudiantado pueda liberarse de cualquier distracción propia de la ejecución para concentrarse en escuchar, 
observar y evaluar. Finalmente, la autograbación en video es, en sí misma, una herramienta que promueve la autorregulación (Feely, 2017).

En ese sentido, Boucher et al. (2017) llevaron a cabo un estudio en el Reino Unido para medir los efectos de la videograbación en 16 guitarristas de música clásica y encontraron que esta no solo hacía reflexionar a los sujetos participantes sobre su manera de tocar, sino también sobre sus procesos de aprendizaje.

Daniel (2001) realizó un estudio que le permitió determinar las percepciones de 35 estudiantes de ejecución musical respecto a la utilización de la videograbación como herramienta de autoevaluación. Confirmó la opinión de McPherson y Zimmerman (2002), pues 72\% de participantes manifestó "nunca" haberse involucrado en un proceso de autoevaluación sobre la base de autograbaciones en video, o haberlo hecho solo "algunas veces". Aun cuando una parte del grupo afirmó haber tenido algunas dificultades para autoevaluarse (la mayoría derivadas de la falta de experiencia), el estudio demostró que la autoevaluación favoreció el desarrollo de las habilidades críticas del pensamiento, las cuales son esenciales para la maduración de las futuras generaciones profesionales de la música. Del mismo modo, la práctica de la autoevaluación, a través del análisis de videos y la autorreflexión, ayudó al estudiantado a independizarse del "gurú" en que llega a convertirse su docente y a tener un criterio más sólido de su propia ejecución.

Es importante destacar que, en los estudios llevados a cabo por Burrack (2002) y Daniel (2001), los sujetos participantes han manifestado sentirse incómodos al autoevaluarse y, en algunos casos, han declarado sentirse avergonzados al escucharse o verse a sí mismos, lo cual podría deberse a factores relacionados con la madurez emocional y psicológica. Sin embargo, ambos estudios reportan un cambio de actitud en sus participantes, tan pronto estos grupos se dieron cuenta de los beneficios que el proceso de autoevaluación brinda.

\section{C) La evaluación de pares}

La evaluación de pares consiste en pedirle a amigos, amigas, compañeras o compañeros que escuchen una ejecución y den retroalimentación. Esta estrategia representa un "probarse" frente a las demás personas (como si fuere un simulacro de recital), y revela el interés por conocer el nivel y calidad de su ejecución desde la perspectiva ajena. El nivel metacognitivo de esta estrategia varía dependiendo del grado de involucramiento de la persona estudiante escuchada. Además, es importante destacar que la evaluación de pares implica el riesgo de recibir comentarios no carentes de prejuicio (Baker-Jordan, 1999). Así, un o una estudiante que se limita a escuchar la retroalimentación de quienes lo observaron y acepta los comentarios sin reparo alguno y sin ejercer su pensamiento crítico, no estará ejerciendo sus habilidades metacognitivas. Por otro lado, quien escucha, reflexiona, compara su propia impresión con la evaluación de sus pares y llega a conclusiones, estará ejerciendo un nivel más alto de metacognición. Este tipo de experiencias contribuye a la maduración de cada estudiante como persona y como futuro o futura profesional de la música, promueve su independización artística e inclusive, puede "inmunizarle" ante comentarios mal intencionados.

El tocar frente a los demás individuos para recibir retroalimentación se relaciona con la tutoría de pares. De acuerdo con Sheldon (2005), la tutoría de pares contribuye al logro, a desarrollar las destrezas para resolver problemas y facilita el entendimiento entre estudiantes. Más aun, la tutoría de pares en contextos de ejecución musical, no solo contribuye a mejorar el nivel del tutorado, sino también el del tutor o la tutora (Alexander y Dorow, 1983).

\section{D) La lista de cotejo}

Crear una lista de cotejo de objetivos a lograr para cada sesión de práctica es una estrategia altamente efectiva. De acuerdo con Cremaschi (2012), autorregularse a través de diarios y bitácoras promueve la reflexión, fomenta la metacognición y suscita el cumplimiento de las tres fases de práctica efectiva (preparación, 
ejecución y autoevaluación de la práctica). Los resultados de una investigación llevada a cabo por Kim (2008), con grupos universitarios de estudiantes de instrumentos de cuerda, revelaron que el uso de un diario de práctica semiestructurado contribuía significativamente en su autorregulación, fomentaba los beneficios indicados por Cremaschi y promovía la concentración.

\section{EL PORTAFOLIO ELECTRÓNICO COMO CATALIZADOR DE LA AUTOEVALUACIÓN ESTUDIANTIL Y LA EVALUACión DEL SU MAESTRO O MAESTRA}

Para efectos de este artículo, se entenderá por portafolio electrónico la recolección intencional, cronológica y sistemática de un conjunto de evidencias relacionadas con objetivos educativo-musicales específicos y predeterminados, que reflejan el proceso de enseñanza y aprendizaje musical del estudiantado. Estas evidencias estarán representadas por archivos y recursos digitales y deben ir acompañadas de comentarios y reflexiones tanto por parte del alumnado como del profesorado. El portafolio electrónico implica reflexionar, analizar, valorar, revisar y evaluar los objetivos planteados, los procesos llevados a cabo para alcanzarlos y los logros obtenidos (Prendes-Espinosa y Sánchez-Vera, 2008; Zúñiga-Benavides; 2007). No es una compilación aleatoria de trabajos, sino que muestra el crecimiento progresivo y los logros obtenidos por cada estudiante en relación con un programa establecido (Díaz-Barriga, 2006).

Las evidencias, reflexiones, análisis, evaluaciones y demás que integran un portafolio electrónico permiten llevar a cabo un proceso de autoevaluación/evaluación de naturaleza cualitativa (aunque es posible añadir criterios cuantitativos), longitudinal y, sobre todo, basada principalmente en el proceso y no en el producto.

Tomando en cuenta que la práctica instrumental representa la parte medular en la formación del músico profesional; que el estudiantado debe autoevaluar los resultados que emanen de esta misma, para poder reiniciar el proceso de aprendizaje de una obra y tener un crecimiento sostenido; y que el maestro o maestra, a su vez, debe evaluar el trabajo estudiantil de una manera objetiva, realista y justa, el autor propone la implementación del portafolio de aprendizaje como instrumento que cataliza la labor de ambos actores del proceso de enseñanza y aprendizaje.

En primer lugar, el portafolio representa en sí mismo una estrategia de autorregulación de alto nivel metacognitivo a través de la cual el estudiantado asume responsabilidad por su propio aprendizaje al monitorizar y evaluar sus propios esfuerzos por medio de la recolección de evidencias que muestren su progreso. El segundo lugar, representa un instrumento que permite una evaluación integral por parte del personal docente a través de la monitorización y revisión de los productos compilados por el estudiantado que reflejan sus esfuerzos, avances y logros.

A través de estrategias didácticas y de una interacción continua, abierta y positiva entre docente y estudiante, se pueden establecer criterios claros y realistas para la conformación del portafolio. Entre otras cosas, cada estudiante podría recopilar evidencias de la manera en que está practicando sus obras, las estrategias que está utilizando para vencer los retos, los criterios que está utilizando para evaluar los resultados y las acciones que está tomando para mejorar su desempeño. Las evidencias podrían estar representadas por registros escritos tales como: reflexiones, bitácoras y diarios elaborados en Word, listas de cotejo elaboradas en Excel, así como registros digitales, tales como: archivos de video y audio de fragmentos de la práctica instrumental y, cuando la tarea lo requiera, gráficas comparativas de Audacity. Por supuesto, el portafolio puede incluir grabaciones en audio, de comentarios de compañeros o compañeras evaluando su desempeño, así como reflexiones, observaciones y sugerencias otorgadas por su docente. El número de posibilidades para crear y organizar las evidencias es grande y el límite lo pondrán el propio estudiantado y su profesorado.

Las páginas de internet como Google Sites, OneDrive y otras similares, y las aplicaciones de video y audio grabación con que hoy en día cuentan los teléfonos celulares, representan recursos electrónicos que se encuentran casi al alcance de todas las personas y que pueden utilizarse para crear un buen portafolio electrónico (Carbajal, 2016). 


\section{Algunos aspectos educativos Que podrían MEJorarse a través DEL uso del PORTAFOLIO DE EVIDENCIAS}

\section{A) La práctica efectiva}

El estudiantado tiende a reflexionar sobre la importancia de los aspectos educativos sobre la base de la evaluación. Si el personal instructor no evalúa un determinado aspecto de la enseñanza, sus estudiantes suelen pensar que este no es importante (Zarzar-Charur, 2006). A través del portafolio de evidencias, el maestro o maestra podría monitorizar y evaluar la práctica de sus estudiantes y retroalimentarles más eficientemente sobre la calidad de esta, con lo cual demostraría la importancia que esta dimensión tiene en el proceso de aprendizaje.

En un estudio que involucró 127 docentes y 134 estudiantes de pregrado, Kostka (2002) encontró que, mientras el $100 \%$ de docentes consideraba que abordaba el tema de las estrategias de práctica efectiva en sus lecciones, solo un $69 \%$ de estudiantes tenía esa percepción. En ese sentido, el portafolio representa un instrumento a través del cual se puede reiterar este tema de una manera clara, objetiva y continua.

\section{B) Énfasis en el proceso, no en el producto}

Es importante destacar que el portafolio electrónico promueve que el estudiantado se concentre en el proceso de aprendizaje (adquisición y perfeccionamiento de determinados conocimientos, habilidades, destrezas y actitudes) y aprecie y valore los logros resultantes del trabajo diario, algo que pierde de vista frecuentemente, al centrarse en lo que falta por hacer, en lugar de celebrar lo que ya se ha logrado. Este fenómeno, muchas veces visto en las escuelas de música a nivel profesional, en muchos casos favorece el desarrollo de estados psicológicos de frustración e inclusive de depresión, que algunas ocasiones conducen a la deserción escolar. En ese sentido, el portafolio electrónico es, desde la perspectiva del autor, una herramienta que permite llevar a cabo una autoevaluación/evaluación holística e integral y, sobre todo, humanista.

\section{C) Comunicación abierta y monitorización continua}

Aun cuando muchos docentes instruyen sobre la manera de practicar para aprender sus piezas, muchas veces sus estudiantes ignoran sus recomendaciones o no las siguen al cien por ciento. Lo anterior fue definido por Flavell (1976) como déficit de producción, en su teoría sobre metacognición, e implica precisamente que el maestro o mastra no puede asumir que cada estudiante seguirá las instrucciones que se le proporcionan. El portafolio podría reducir este déficit y promover una monitorización continua y una comunicación más honesta y directa entre docente y estudiante.

\section{D) Responsabilidad}

Jorgensen (2000) afirma que, incluso a nivel de educación superior, no todo el estudiantado quiere aceptar la responsabilidad. El portafolio electrónico, como estrategia de autorregulación, fomenta la responsabilidad y lo confronta consigo mismo. 


\section{E) Conciliación de enfoques educativos}

El portafolio electrónico concilia el enfoque pedagógico centrado en la enseñanza (enfoque favorito de muchos maestros y maestras de instrumento) con el enfoque centrado en el aprendizaje. Al mismo tiempo, favorece que el personal docente tenga una visión más amplia y profunda de lo que el estudiantado sabe y puede hacer, refleja los avances en los aprendizajes conceptuales, actitudinales y procedimentales y les permite a docente y estudiante monitorizar los esfuerzos, evaluar los resultados y modificar la práctica con el objetivo de promover una mejora constante.

\section{RECOMENDACIONES}

En años recientes ha habido un interés creciente sobre el aspecto de la evaluación del desempeño en la música (Capistrán-Gracia y Correa-Ortega, 2017). Aun cuando se ha dado gran relevancia a la validez y confiablidad de los instrumentos de evaluación y se han desarrollado estudios sobre el diseño e implementación de rúbricas, las investigaciones han revelado que, en materia de educación de las artes, las evaluaciones tienden a favorecer aspectos subjetivos tales como la expresividad y la proyección. Como consecuencia de esta subjetividad, las evaluaciones suelen estar rodeadas de controversia (Navarro, 2013). En ese sentido, Chacón-Solís (2012) refiere cómo, en un examen, un sinodal había criticado la falta de musicalidad de un estudiante. Sin embargo, cuando este le preguntó cómo podía ser musical, el sinodal se vio incapaz de articular una respuesta.

En otros contextos, la evaluación suele concentrarse en aspectos tales como la asistencia y la participación en lugar de enfocarse en el aprendizaje estudiantil (Pellegrino, Conway y Russel, 2015). Lo anterior parece ser característico en la evaluación de los grandes ensambles musicales, en los que muchas veces quien dirige el grupo evalúa su desempeño en su contexto general, sin quedar claro cuál fue el logro o calidad del desempeño de cada integrante. Así, en un estudio llevado a cabo en un conservatorio de música en Australia, Harrison, Lebler, Carey, Hitchcock y O'Bryan (2013) encontraron que tanto docentes como estudiantes pensaban que la participación en ensambles musicales era de gran importancia para el desarrollo de un músico o una música profesional. Sin embargo, consideraban que los procedimientos de evaluación no siempre eran compatibles con esta meta y que la manera de evaluar al ensamble era confusa y carecía de transparencia. El autor considera que, dada la complejidad de la evaluación de ensambles musicales, una modalidad simplificada del portafolio electrónico podría contribuir a llevar a cabo una evaluación más realista, objetiva, justa, transparente e individualizada.

\section{Epílogo}

A finales del 2015, el autor llevó a cabo un estudio en el que participaron 252 estudiantes de licenciatura en música con énfasis en ejecución, de seis universidades de la Región Centro Occidente de la ANUIES. El estudio reveló que el uso de estrategias de autoevaluación de alto nivel metacognitivo se encontraba en las categorías de "algunas veces" y "rara vez" con una media de 1.86 sobre 4.0 (Capistrán, 2017).

Tradicionalmente, la parte correspondiente al proceso de aprendizaje de instrumento es evaluado sobre la base de la apreciación subjetiva del maestro o de la maestra, ya que como vimos, la mayor parte de la práctica es llevada a cabo por estudiantes en su cubículo. El autor exhorta a docentes y estudiantes a establecer procesos de enseñanza y aprendizaje que involucren evaluaciones serias y responsables en sus modalidades de diagnóstica, continua y final. Especialmente, les exhorta a implementar el portafolio de aprendizaje, pues aparte de desarrollar una actitud metacognitiva en el estudiantado que le lleve a hacerse cargo de su propio aprendizaje, le conduce al logro e impulsa valores como la responsabilidad, la justicia, la integridad, la 
confianza hacia su maestro o maetra y el respeto. Además, coloca a ambos actores en posición de poder rendir cuentas sobre su desempeño, delimita su responsabilidad y fomenta la armonía y el crecimiento musical.

Hasta donde el autor ha investigado, la implementación del portafolio de aprendizaje en la formación de estudiantes de música a nivel profesional ha sido escasamente reportada. Adicionalmente, implica una mayor inversión de tiempo, tanto por la parte docente como estudiantil. Sin embargo, los beneficios superan por mucho cualquier desventaja.

Durante el proceso de aprendizaje, profesorado y alumnado deberán enfrascarse en un intenso trabajo que no solo habrá de producir logros académico-musicales, sino también desarrollar valores humanos. Como explica Gervilla Castillo (2003, p. 98):

Una de las contradicciones de nuestra sociedad de bienestar es la infravaloración de todo esfuerzo, sin el cual no es posible, en múltiples ocasiones, alcanzar valores en alza, tales como la libertad, la autonomía, el autodominio, la solidaridad o la tolerancia. Contradicción que los educadores hoy hemos de saber mostrar y demostrar, pues no es posible alcanzar la meta despreciando el camino que conduce a ella.

La lección de instrumento es mucho más que ir monitorizando el aprendizaje de una obra por parte de nuestros discípulos o discípulas. Representa todo un trabajo de diagnóstico, de planeación y programación, de evaluación continua, de modificación de objetivos, y de constante retroalimentación. El objetivo final es que el estudiantado aprenda a aprender (Hallam, 2001; Jorgensen, 2000; Ross, 2006) desarrolle estrategias de práctica efectiva (Hallam, 1995 y 1997; Hallam, Rinta, Varvarigou, Creech, Papageorgi, Gomes, y Lanipekun, 2012; Nielsen, 2001; Pintrich y Groot, 1990) y logre un crecimiento musical sostenido (Sloboda, 1990). Toca a cada docente y a cada estudiante responsabilizarse y trabajar diligentemente en equipo para lograrlo.

\section{ReFERENCIAS}

Alexander, L. y L. G. Dorow. (1983). Peer tutoring effects on the music performance of tutors and tutees in beginning band classes. Journal of Research in Music Education, 31(1), 33-47.

Baker-Jordan, M. (1999). What are the pedagogical and practical advantages of "three or more" teaching? Pedagogy Saturday III, 22-23. Cincinnati: Music Teachers National Association.

Boucher, M., Dube, F. y Creech, A. (2017). The effect of video feedback on the self-assessment of a music performance by college level classical guitarists. En G. Hughes (Ed.), Ipsative assessment and learning gains: Case studies from international practitioners (pp. 197-220). Basingstoke, Hampshire: Palgrave Macmillan.

Burrack, F. (2002). Enhanced assessment in instrumental programs. Music Educators Journal, 88(6), 27-32.

Byo, J. y Cassidy, J. W. (2008). An exploratory study of time use in the practice of music majors self-report and observation analysis. Update: Applications of Research in Music Education, 27(1), 33-40.

Capistrán-Gracia, R. W. (Julio, 2014). Enseñanza, implementación y evaluación de técnicas y estrategias de práctica diaria del instrumento musical. Ponencia presentada en el XX Seminario Latinoamericano de Educación Musical. Heredia, Costa Rica.

Capistrán-Gracia, R. W. y Correa-Ortega, J. P. (2017). La evaluación del desempeño musical y el uso de rúbricas. Docere, 8(17), 28-34.

Capistrán-Gracia, R.W. (2017). La práctica musical efectiva. Revisión de literatura, resultados de investigación y propuesta metodológica. Aguascalientes, México: Editorial UAA.

Carbajal-Vaca, I. S. (2016). El portafolio electrónico: La Experiencia en programas de formación musical en el pregrado. Ponencia presentada en la V Semana Internacional de Educación Artística. Universidad Autónoma de Chihuahua.

Chacón-Solís, L. (2012). ¿Qué significa "evaluar" en música? Revista Electrónica Complutense de Investigación en Educación Musical, 9, 1-25. 
Raul Wenceslao Capistrán Gracia[1]. El portafolio electrónico como instrumento de evaluación y com...

Cremaschi, A. (2012). The effect of a practice checklist on practice strategies, practice self-regulation and achievement of collegiate music majors enrolled in a beginning class piano course. Research Studies in Music Education, 34(2), 223-233.

Daniel, R. (2001). Self-assessment in performance. British Journal of Music Education. 18(3), 215-226.

Díaz-Barriga, F. (2006). Enseñanza situada: Vínculo entre la escuela y la vida. México: McGraw-Hill.

Fautley, M. (2013). The potential of audio and video for formative assessment purposes in music education in the lower secondary school in England: Issues arising from a small-scale study of trainee music teachers. Journal of Music, Technology \& Education, 6(1), 29-42.

Feely, P. K. (2017). The Effects of Video Recording on the Level of Expertise and Self-Regulated Learning Ability of Adults in a Beginner Classical Guitar Class (Tesis de maestría). The University of Western Ontario, London, Ontario, Canada.

Flavell, J. H. (1976). Meta-cognitive aspects of problem solving. En L. B. Resnick (Ed.), The Nature of Intelligence (pp. 231-235). Mahwah, NJ: Erlbaum.

Garrison, L. (2009/2010). Audio recording as a teaching tool in applied music. College Music Symposium, 49/50, 284-286.

Gaunt, H. (2008) One-to-one tuition in a conservatoire: the perceptions of instrumental and voice teachers. Psychology of Music, 36, 215-245.

Gervilla, E. (2003). Pedagogía del esfuerzo y cultura del placer. Revista Española de Pedagogía, 224, 97-114.

Hallam, S. (1995). Professional musician's approaches to the learning and interpretation of music. Psychology of Music, 23, 111-128.

Hallam, S. (1997). What do we know about practising? Towards a model synthesising the research literature. En H. Jorgensen y A. Lehman (Eds.), Does Practice Make Perfect? Current Theory and Research on Instrumental Music Practice (pp. 179-231). Oslo: Norges musikkhogskole Publikasjoner.

Hallam, S. (2001). The development of meta-cognition in musicians: Implications for education. The British Journal of Music Education, 18(1), 27-39.

Hallam, S., Rinta T., Varvarigou, M., Creech, A. Papageorgi, L., Gomes, T., y Lanipekun, J. (2012). The development of practising strategies in young people. Psychology of Music, 40(5), 652-680.

Harrison, S. D., Lebler, D., Carey, G., Hitchcock, M. y O'Bryan, J. (2013). Making music or gaining grades? Assessment practices in tertiary music ensembles. British Journal of Music Education, 30(1), 27-42.

Jorgensen, H. (2000) Student learning in higher instrumental education: Who is responsible? British Journal of Music Education, 17, 67-77.

Jorgensen, H. (2004). Strategies for individual practice. En Williamon, A. (Ed.), Musical Excellence: Strategies and Techniques to Enhance Performance (pp. 85-104). Oxford: Oxford University Press.

Kim, S. J. (2008). Using a practice diary to promote self-regulated instrumental practice. American String Teacher, 58(3), 34-36.

Kostka, J. (2002). Practice expectations and attitudes: A survey of college-level music teachers and students. Journal of Research in Music Education, 50, 145.

Lundin, R. A. (1967). An Objective Psychology of Music. New York: The Ronald Press Company.

McPherson, G. E. y Renwick, J. M. (2001). A longitudinal study of self-regulation in children's musical practice. Music Education Research, 3(2), 169-186.

McPherson, G. E. y Zimmerman, B. J. (2002). Self-regulation of musical learning: A social cognitive perspective. In R. Colwell y C. Richardson (Eds.), The New Handbook of Research on Music Teaching and Learning: A Project of the Music Educators National Conference (pp. 327-347). New York: Oxford University Press.

McPherson, G. y McCormick, J. (1999). Motivational and self-regulated learning components of musical practice, Bulletin of the Council for Research in Music Education 17(141), 98-102.

Navarro, J. (2013). La evaluación musical en una escuela universitaria mexicana: Opiniones del alumnado. Revista Electrónica de Música en la Educación, 32, 19-52. 
Nielsen, S. (1999). Learning strategies in instrumental music practice. British Journal of Music Education, 16(3): 275-291.

Nielsen, S. (2001) Self-regulating learning strategies in instrumental music practice. Music Education Research, 3(2), 155-167.

Pellegrino, K., Conway, C.M. y Russel, J.A. (2015). Assessment in performance-based secondary music classes. Music Educators Journal, 9, 48-55.

Pintrich, P. R., y De Groot, E. V. (1990). Motivational and self-regulated learning components of classroom academic performance. Journal of Educational Psychology, 82(1), 33-40.

Pitts, S., Davidson, J.W.y McPherson, G.E. (2000). Developing effective practise strategies: Case studies of three young instrumentalists. Music Education Research, 2(1), 45-56.

Prendes-Espinosa, M. P. y Sánchez-Vera, M. M. (2008). Portafolio electrónico: Posibilidades de los docentes. Pixel-Bit. Revista de Medios y Educación, 32, 21-34. Recuperado de http://www.redalyc.org/articulo.oa?id=36803202

Rosenthal, R., Durairaj, M. y Magann, J. (2009). Musicians' descriptions of their expressive musical practice. Bulletin of the Council for Research in Music Education, 181(4), 37-49.

Ross, J. A. (2006). The reliability, validity, and utility of self-assessment. Practical Assessment Research \& Evaluation, 11(10), 1-13.

Sheldon, D. A. (2005). Peer and cross-age tutoring in music. Journal of Research in Music Education, 53(1), 40-50.

Silveira, J. y Gavin, R. (2015). The effect of audio recording and playback on self-assessment among middle school instrumental music students. Psychology of Music, 1-13.

Sloboda, J. (1990). Musical excellence-How does it develop? En Howe, M. J. A. (Ed.), Encouraging the Development of Exceptional Skills and Talents. Leicester: British Psychological Society.

Sloboda, J. A., Davidson, J. W., Howe, M. J. A. y Moore, D. G. (1996). The role of practice in the development of performing musicians. British Journal of Psychology, 87, 287-309.

Zarzar-Charur, C. A. (2006). Habilidades básicas para la docencia. México: Grupo Patria Cultura.

Zimmerman, B. J. (1986). Becoming a self-regulated learner: Which are the key subprocesses? Contemporary Educational Psychology, 11, 307-313.

Zimmerman, B. J. (2008). Investigating self-regulation and motivation: Historical background, methodological developments, and future prospects. American Educational Research Journal, 45(1), 166 -183.

Zúñiga-Benavides, J. F. (2007). El portafolio electrónico: Una opción metodológica. Bibliotecas. 25(2), 27-39.

\section{Notas}

[1]

Pianista, educador e investigador mexicano. Recibió la licenciatura en ejecución pianística en la Escuela Superior de Música y Danza de Monterrey, la Maestría en Ejecución Pianística con distinción en Baylor University y el doctorado en Pedagogía Pianística en Texas Tech University. Al graduarse recibió el reconocimiento de la Music Teachers National Association como alumno distinguido, así como el reconocimiento de la Universidad de Texas Tech como maestro sobresaliente. Recientemente llevó a cabo una estancia de investigación en el Institute of Education de la University College London. En dos ocasiones (1994 y 2005) ha sido distinguido con becas otorgadas por el Programa de Estímulo a la Creación y al Desarrollo Artístico de Tamaulipas para la realización de proyectos de rescate del patrimonio cultural. En la actualidad trabaja como profesor investigador titular de la Universidad Autónoma de Aguascalientes en donde representa el Doctorado Interinstitucional en Arte y Cultura.

\section{BY-NC-ND}

